\title{
Raising Awareness of Mental Health in Higher Education (RAMHHE) Research Activity for Educators
}

\section{Josephine NwaAmaka Bardi, MSc, RMN}

Economic and Social Research Council (ESRC) PhD Student, University of Nottingham, School of Health Sciences, Queens Medical Centre, Nottingham, United Kingdom, NG7 2UH

Authors Affiliation: London School of Economics and Political Science, University of East London

Globally, there has been increasing concern about university students' reporting mental health conditions, yet their mental health needs remain mostly unaddressed. This Teaching Tip introduces Raising Awareness of Mental Health in Higher Education (RAMHHE), a student-centred and pedagogical research activity that adopts qualitative methods of interviews and focus group discussions to explore the meaning and understanding of mental health among university students. The RAMHHE research activity enables collaborative dialogue about mental health between students and educators, using four questions: (1) What is mental health? (2) Who would you speak to about your mental health? (3) How is mental health viewed in your community and (4) How can we support students with mental health? The first question will provide an understanding of the student's definition of mental health and how this differs from the educator's definition; the second question will support a range of referral pathways for students. The third question is useful for understanding the varied meanings of mental health among student's students from diverse cultural and religious backgrounds because students may not seek help if their culture or religion does not recognise mental health. The final question encourages an alluniversity dialogue on how to support students with mental health conditions. The use of focus group discussions will enable students to share interpretations and meanings. Findings will prompt the development of student-informed mental health interventions and may positively impact on student access, retention, attainment and progression outcomes. A figure of the model is in Supplemental Digital Content. 


\section{Conceptual Model: RAMHHE Research Activity for Educators}

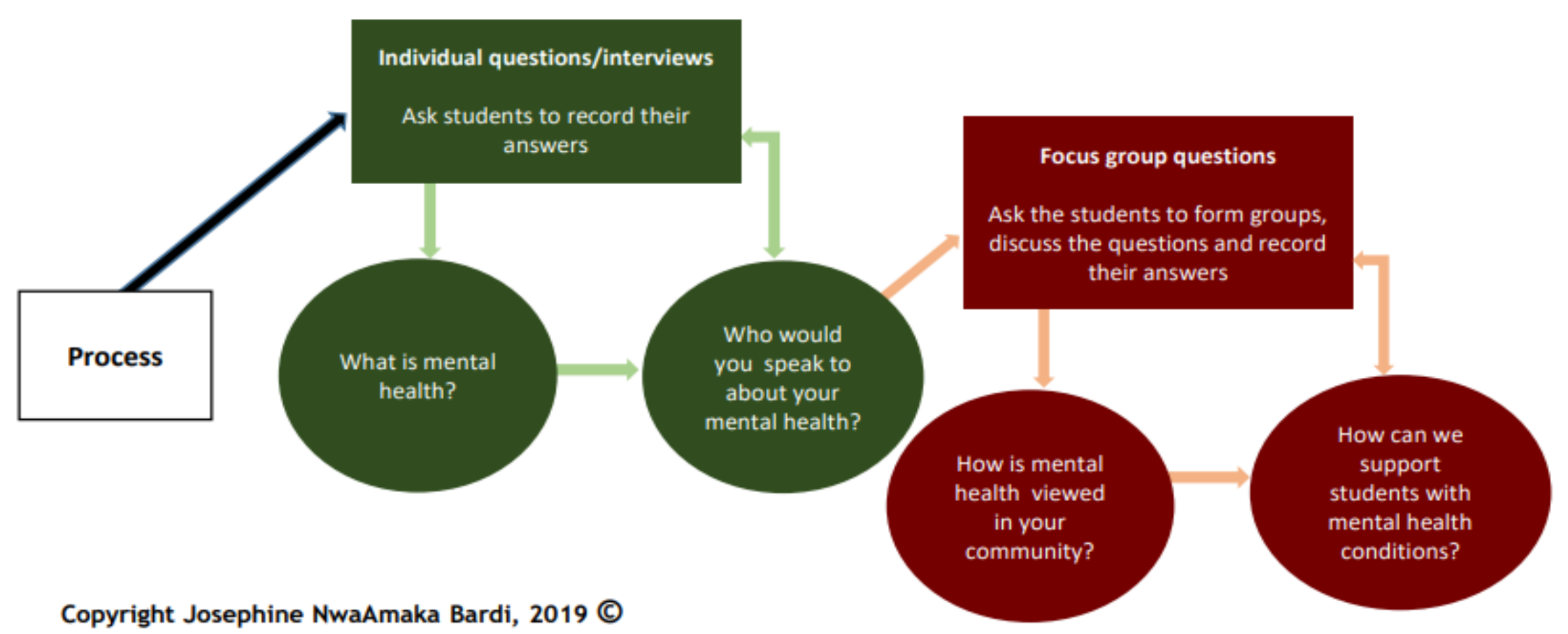

Reprint and reuse by permission only 